\title{
Cyclic Estrogen Replacement Improves Cognitive Function in Aged Ovariectomized Rhesus Monkeys
}

\author{
Peter R. Rapp, ${ }^{1}$ John H. Morrison, ${ }^{1}$ and Jeffrey A. Roberts ${ }^{2}$ \\ ${ }^{1}$ Kastor Neurobiology of Aging Laboratories, Fishberg Research Center for Neurobiology, Department of Geriatrics and Adult Development, Mount Sinai \\ School of Medicine, New York, New York 10029-6574, and ${ }^{2}$ California National Primate Research Center, University of California, Davis, Davis, California \\ 95616-8615
}

\begin{abstract}
Among the identified risks and benefits of hormone-replacement therapy, the effects of treatment on cognitive function in postmenopausal women have proved difficult to define. Here we conducted a controlled, prospective analysis in a nonhuman primate model to test whether surgical menopause and estrogen replacement influence the cognitive outcome of normal aging. Sixteen aged rhesus monkeys were ovariectomized, and throughout the course of subsequent neuropsychological assessment, half received a regimen of low-dose, cyclic estradiol replacement. Hormone treatment substantially reversed the marked age-related impairment vehicle-injected monkeys exhibited on a delayed response test of spatial working memory. Modest improvement was also observed on a delayed nonmatching-tosample recognition memory task. In contrast, ovariectomy exacerbated age-related deficits in object discrimination learning; the magnitude of this effect was equivalent among vehicle- and estrogen-treated monkeys. Together, these results demonstrate that ovarian hormone status can broadly influence normal cognitive aging in monkeys, affecting capacities mediated by multiple brain regions, including the prefrontal cortex and the medial temporal lobe memory system. The animal model established here should enable progress toward defining the neurobiological mechanisms that mediate the beneficial effects of estrogen on age-related cognitive decline in primates.
\end{abstract}

Key words: menopause; hormone replacement; cognitive aging; monkey; hippocampus; prefrontal cortex

\section{Introduction}

Life expectancy has increased dramatically since 1900, and women in many industrialized countries now live nearly half of their adult lives after menopause. The significance of this demographic shift is underscored by evidence that the regulatory effects of ovarian hormones extend well beyond the neuroendocrine reproductive axis, affecting the structure and function of a variety of extrahypothalamic brain regions that are critical for normal cognition (McEwen, 2002). Although these findings have prompted substantial speculation that estrogen deficiency at menopause might modulate the course of age-related cognitive decline, designing a compelling test of this proposal in women has proved challenging (Hogervorst et al., 2000). Research on the cognitive effects of hormone-replacement therapy (HRT), for example, is complicated by the difficulty of matching treatment and control groups according to chronological age, education, overall health status, and other subject characteristics that can

Received Feb. 25, 2003; revised April 24, 2003; accepted April 24, 2003

This work was supported National Institutes of Health Grants AG10675 and AG10606 and California National Primate Research Center Base Grant RR-000169. We thank Harry Arnell, Chad Ellis, Mary Roberts, and Heather McKay for expert technical assistance; Dr. Bill Lasley and members of the endocrinology core facility at the California National Primate Research Center for ovarian hormone assays; the veterinary and animal care staff at the California National Primate Research Center; and Drs. Michela Gallagher and Bruce McEwen for discussion and editorial comments.

Correspondence should be addressed to Dr. Peter R. Rapp, Kastor Neurobiology of Aging Laboratories, Mount Sinai School of Medicine, Box 1639, One Gustave L. Levy Place, New York, NY 10029-6574. E-mail: peter.rapp@mssm.edu.

Copyright $\odot 2003$ Society for Neuroscience $\quad$ 0270-6474/03/235708-07\$15.00/0 influence neuropsychological function independent of ovarian hormone status. Information about additional critical variables (such as the specific formulation, dose, and duration of HRT; compliance with therapy; and the efficacy of treatment in terms of circulating hormone levels) is also frequently unavailable or difficult to verify by observational and epidemiological investigation. Given these limitations, it may not be surprising that studies of ovarian hormone influences on cognitive aging in women have reported conflicting results (Barrett-Connor and KritzSilverstein, 1993; Resnick and Maki, 2001; Sherwin, 2002).

Here we adopted a controlled, prospective design in a nonhuman primate model to test the effects of ovariectomy and estrogen replacement on normal cognitive aging. Monkeys selected for inclusion were premenopausal or perimenopausal before surgery and averaged 22 years of age, approximately comparable with 55- to 65-year-old women (Tigges et al., 1988). Like healthy women in this age range, rhesus monkeys in their early 20 s typically exhibit only mild impairments in memory and other neuropsychological domains (Bachevalier et al., 1991). Thus, our aim in focusing on this early segment of the aging process was to examine the influence of estrogen manipulation when cognitive function is vulnerable to decline, but before the onset of pronounced impairment that might be insensitive to rescue. It is also noteworthy that, because rhesus monkeys do not develop Alzheimer's disease, our approach permitted an evaluation of estrogen effects specifically on normal cognitive aging, unconfounded by dementing illness. Finally, in contrast to rats and mice, the tem- 

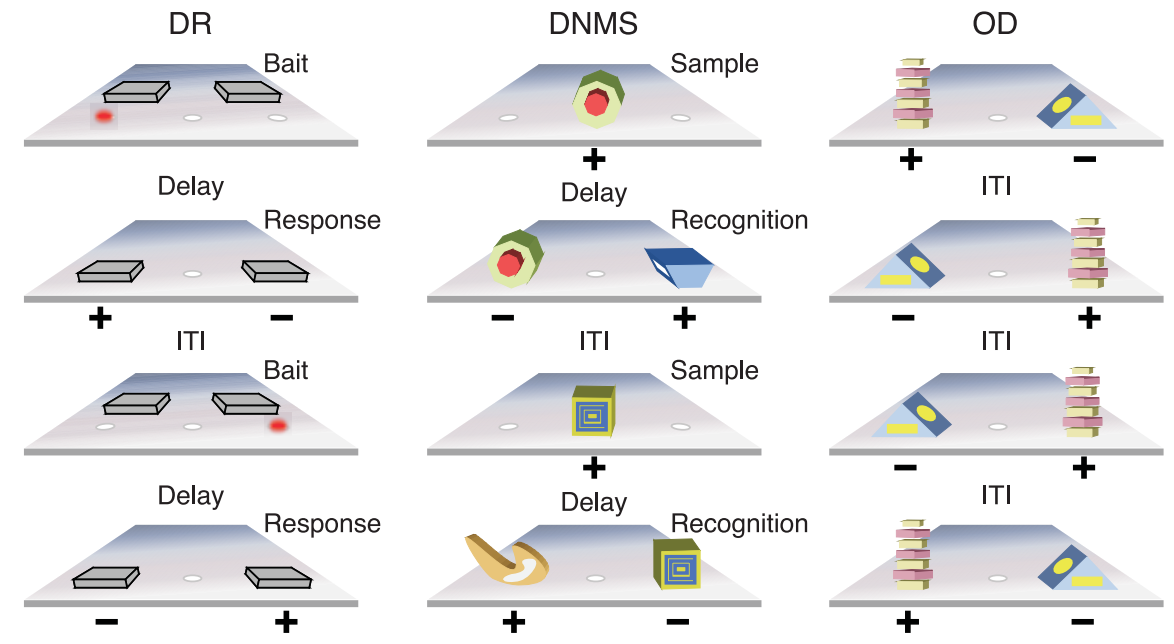

Figure 1. Schematic representation of the neuropsychological test battery. Left, DR; red dot signifies food reward; middle, DNMS; and right, two-choice 0D. +, Baited; - , unbaited.

poral dynamics of normal menstrual cyclicity, and the late-life onset of menopause, are closely similar in female rhesus monkeys and women (Gilardi et al., 1997). These considerations, supplemented by a long history of neuropsychological research in monkeys, guided our selection of a nonhuman primate model for defining the influence of ovarian hormone decline on cognitive aging.

\section{Materials and Methods}

Subjects and hormonal screening. Sixteen aged female rhesus monkeys served as subjects (Macaca mulatta; mean age \pm SEM, 22 years \pm 7 months). Animals were naive with respect to formal memory testing and had no physical disabilities or experimental histories that might confound interpretation of the behavioral results (e.g., chronic dietary or pharmacological manipulation). Subjects were singly housed in colonies of $\sim 40$ monkeys. Water was available ad libitum in the home cage, and standard rations of monkey chow were provided daily after behavioral testing, supplemented regularly with fresh fruit.

Before the experiments, candidate animals were screened according to reproductive history, menses activity over the preceding year, and urinary hormone profiles sampled daily over 3 months. Urine samples were collected noninvasively, and excretion of estrone conjugates and pregnanediol-3-glucuronide (metabolites of estrogen and progesterone, respectively) was measured by enzyme immunoassay (Shideler et al., 1990), using an approach validated for tracking ovarian hormone senescence in aged monkeys (Gilardi et al., 1997). On the basis of these established criteria, monkeys classified as premenopausal and perimenopausal were selected for behavioral assessment. Serum estradiol measured during the course of the experiments was determined by standard radioimmunoassay (Korenman et al., 1974).

For certain analyses, results from the aged ovariectomized (OVX) monkeys were also compared with performance in intact young adults ( $n=23$; mean age \pm SEM, 5.2 years \pm 5 months). Behavioral assessment in these subjects was conducted using the same apparatus, task stimuli, and training protocols as for the aged animals. Like the OVX groups, the young monkeys were naive with respect to formal behavioral testing before the present assessment, and all conditions of housing and maintenance were similar. Although young females $(n=15)$ and males $(n=$ 8 ) were studied, there were no reliable sex differences in performance, and the pattern of results was the same when males were excluded from consideration. Four of the young intact animals were tested on the delayed response (DR) procedure only; therefore, the young group consisted of 19 monkeys for the delayed nonmatching-to-sample (DNMS) and object-discrimination tasks (described below). All experimental procedures were approved by Institutional Animal Care and Use Committees at the University of California, Davis and the Mount Sinai School of
Medicine and conformed to other institutional and National Institutes of Health guidelines.

Ovariectomy and estrogen replacement. The aged monkeys received bilateral ovariectomies before neuropsychological testing. After the administration of ketamine $(10 \mathrm{mg} / \mathrm{kg}, \mathrm{i} . \mathrm{m}$.) and atropine $(0.04 \mathrm{mg} / \mathrm{kg}$, s.c.), animals were intubated and placed on isoflurane anesthesia. A ventral midline incision was effected, and after direct visualization of the ovaries, the ovarian vessels and fallopian tubes were isolated, ligated, and severed. The ovaries were subsequently removed and the vessels observed for bleeding. The abdominal wall was closed in layers, animals were observed until responsive, and oxymorphone was provided for postoperative analgesia $(1.5 \mathrm{mg} / \mathrm{kg}$, i.m., 3 times $/ \mathrm{d}$ for $2 \mathrm{~d})$.

Beginning an average of $30 \pm 1.7$ (mean \pm SEM) weeks after surgery, eight randomly assigned monkeys received estradiol cypionate (100 $\mu \mathrm{g} / 1 \mathrm{ml}$ of sterile peanut oil, i.m.; Pharmacia, Peapack, NJ) in a single injection every 3 weeks (group OVX-E), and the remaining, agematched subjects were provided equivalent volume vehicle injection according to the same schedule (group OVX-veh; $n=8$ ). Estrogen and vehicle injections were coded and administered in a blinded manner. The OVX-to-treatment interval allowed extensive postoperative hormone monitoring and ensured that transient hormonal dynamics after surgery stabilized before behavioral assessment. In comparison with the conjugated equine estrogen (CEE) formulations commonly prescribed in clinical practice in the United States, which predominantly comprise estrone sulfate, the active estrogen in the regimen used here was estradiol. Relative to CEEs, estradiol delivered intramuscularly exhibits greater bioavailability and estrogen-receptor activity (O'Connell, 1995).

Behavioral testing. Neuropsychological assessment was conducted in a manual apparatus by experimenters blinded with respect to the treatment condition of the subjects. All behavioral testing, in both the aged OVX groups and young intact monkeys, was performed at the California National Primate Research Center, Davis, California. Tasks were administered in the order listed using protocols described in detail previously (Rapp, 1990; Rapp and Amaral, 1991; O’Donnell et al., 1999). For all tasks, a one-way mirror hid the experimenter from view when monkeys responded, and a white-noise generator was used to mask extraneous sounds.

$D R$. Trials in this test of visuospatial working memory consisted of baiting and response phases (Fig. 1, left). Subjects watched from behind a transparent screen while one of the lateral wells of the apparatus was baited with a food reward, and both wells were then covered with identical plaques. During initial training, the screen was raised immediately, allowing monkeys to displace a plaque and retrieve the reward if the baited location was selected. Testing continued in this manner until animals met a criterion of $90 \%$ correct or better across 9 consecutive blocks of 10 trials. Subsequent testing was similar except that a $1 \mathrm{sec}$ retention interval was imposed between baiting and the opportunity to respond. Delays were implemented by lowering an opaque screen between the subject and the reward wells of the apparatus. Training with a 1 sec delay continued to the same performance criterion ( $\geq 90 \%$ correct over 90 trials), and the demands of testing were then made progressively more challenging by imposing successively longer retention intervals of 5, 10, 15, 30, and $60 \mathrm{sec}$. Each delay was tested for a total of 90 trials [30 trials/daily session; intertrial interval (ITI) $=20 \mathrm{sec}]$. For all tasks including DR, the left and right wells were baited equally often across trials within a test session, disallowing a simple rule-based task solution (e.g., go left).

DNMS. DNMS trials consisted of a sample object presentation followed by a recognition test (Fig. 1, middle). The sample appeared over the baited central well of the test tray, and after a response, the opaque barrier was lowered to impose the retention interval. The sample item was subsequently presented together with a novel object that covered a 


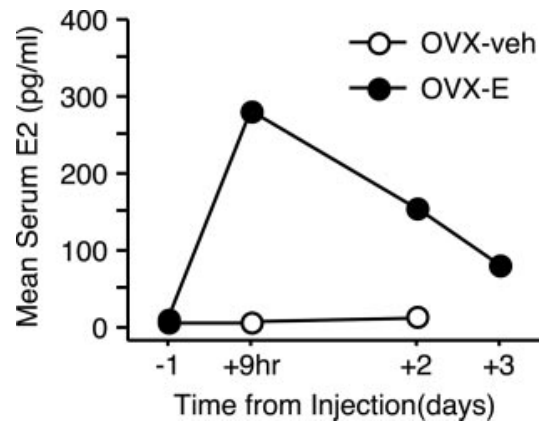

Figure 2. Mean serum estradiol (E2) levels in aged OVX monkeys $1 \mathrm{~d}$ before, and $9 \mathrm{hr}, 2 \mathrm{~d}$, and $3 \mathrm{~d}$ after vehicle or hormone injection. The results include data from every monkey, but not all time points were evaluated in each subject.

reward. Objects were drawn from a large pool such that a new pair was presented on each trial. During initial testing, monkeys learned the nonmatching rule with a $10 \mathrm{sec}$ retention interval, to a criterion of $90 \%$ correct or better (across 100 trials, 20 trials/d, ITI $=30 \mathrm{sec}$ throughout testing). Demands on recognition memory were subsequently increased by imposing successively longer delays of $15,30,60$, and $120 \mathrm{sec}(100$ trials total at each delay, 20 trials/d) and $600 \mathrm{sec}$ (50 trials total, 5 trials/d). Monkeys remained in the test chamber during all retention intervals.

Two-choice object discrimination. On each object discrimination (OD) trial, subjects chose between two visually distinct objects, one of which was consistently associated with reward across trials (Fig. 1, right). The same discrimination problem was presented for 30 trials/d across 2 consecutive days, followed $48 \mathrm{hr}$ later by a final 30-trial session (ITI $=15$ sec); animals were tested on four successive discriminations according to this schedule.

\section{Results}

The coordinated fluctuation of urinary estrone conjugates and pregnanediol observed in intact animals was disrupted after ovariectomy, serum estradiol levels declined to near undetectable levels, and regular menstrual bleeding was eliminated. Hormone injection produced a sharp rise in serum estradiol that declined toward preinjection baseline values over several days (Fig. 2). During this period, peak circulating estrogen levels were comparable with preovulatory values observed in intact females (Shideler et al., 1990) and substantially higher than in the OVXveh group. The bioefficacy of hormone replacement was also confirmed by the observation that all OVX-E monkeys exhibited instances of breakthrough bleeding after treatment, whereas similar effects could not be verified in OVX-veh animals.

\section{Cognitive effects of ovariectomy and cyclic estrogen replacement}

Behavioral testing was initiated $2 \mathrm{~d}$ after the second estradiol or vehicle injection using a DR task (Fig. 1). This well characterized test of spatiotemporal working memory requires the functional integrity of the dorsolateral prefrontal cortex (Mishkin and Pribram, 1956; Goldman-Rakic, 1987; Fuster, 2001) and is a particularly sensitive marker of age-related cognitive decline in monkeys (Rapp and Amaral, 1989; Bachevalier et al., 1991).

One OVX-veh monkey failed to reach the DR performance criterion at the $0 \mathrm{sec}$ delay within the limits of testing (1200 trials) and scored near chance levels of accuracy throughout training. Therefore, DR results from this animal were excluded from analysis. The remaining hormone-replacement and control subjects learned the DR procedure with a 0 sec delay at nearly identical rates (ANOVA; $p>0.9$ ), demonstrating that both groups were motivated to perform and were capable of high levels of accuracy
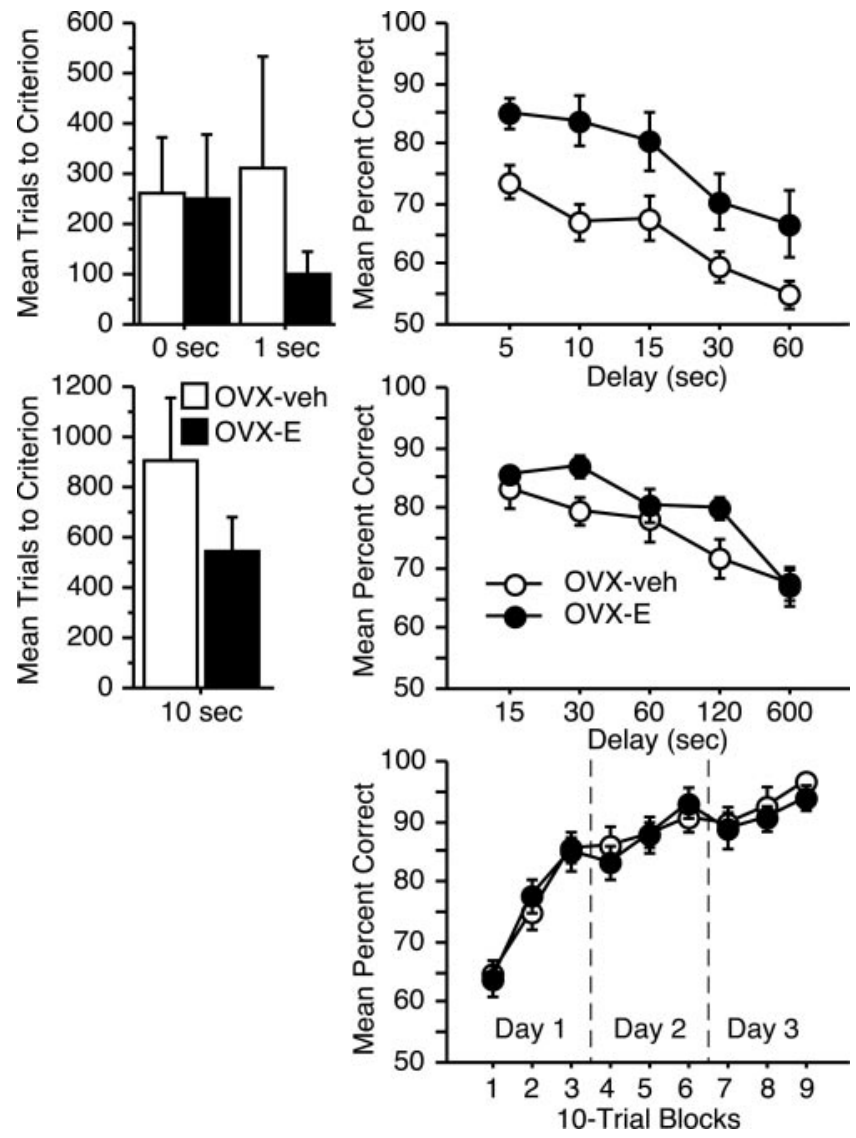

Figure 3. Neuropsychological performance in aged OVX-veh and OVX-E monkeys. Top, Mean number of trials required to reach the acquisition criterion on the DR task at 0 and $1 \mathrm{sec}$ delays (left), and mean percent correct across delays of 5-60 sec (right). Middle, Mean trials required to learn the DNMS procedure with a 10 sec delay (left), and mean percent correct across retention intervals of 15-600 sec (right). Bottom, Mean percent correct across 10 -trial blocks of object discrimination learning (days 1 and 2), and retention after $48 \mathrm{hr}$ (day 3 ). With the exception of one case in which an OVX-E animal completed only three problems, scores for individual animals were averaged across four discriminations. Error bars indicate SEM.

(Fig. 3, top left). Although group OVX-E required only a third as many trials as OVX-veh monkeys to relearn the task with a $1 \mathrm{sec}$ delay, this difference was not statistically reliable because of variability among the controls $(p>0.3)$.

In contrast to their overlapping performance during acquisition, aged OVX monkeys given estrogen replacement scored significantly better than group OVX-veh when successively longer retention intervals were imposed (repeated-measures ANOVA; main group effect: $F_{(1,13)}=9.2, p<0.01$ ) (Fig. 3, top right). The magnitude of this benefit was comparable across short and long delays (group by delay interaction: $p>0.8$ ). Scores for both groups declined as the retention interval increased (main effect of delay: $\left.F_{(4,52)}=14.6, p<0.0001\right)$, demonstrating that the procedure effectively taxed memory. We previously reported a similar pattern of superior DR performance in aged ovary-intact premenopausal monkeys relative to age-matched perimenopausal/ postmenopausal females (Roberts et al., 1997). Thus, descriptive and experimental data converge on the conclusion that estrogen status in monkeys significantly regulates the cognitive outcome of aging as assessed by DR.

To further define the scope of the influence of estrogen on cognitive aging, subjects were tested next on DNMS (Fig. 1). Recognition memory measured by this task requires the hippocampal formation and perirhinal cortex (Mishkin, 1978; 
Squire and Zola-Morgan, 1991), but is relatively spared after dorsolateral prefrontal cortex lesions that cause severe DR impairment (Bachevalier and Mishkin, 1986). DNMS performance is also susceptible to age-related decline in monkeys (Presty et al., 1987; Moss et al., 1988), although there is considerable variability across individuals and a substantial number score as accurately as young adults (Rapp and Amaral, 1991).

On average, group OVX-E learned the DNMS procedure in substantially fewer trials than age-matched OVX controls, but this numerical difference was not statistically reliable $(p>0.2)$ (Fig. 3). Recognition accuracy declined in both treatment conditions across successively longer retention intervals (repeatedmeasures ANOVA; main effect of delay: $F_{(4,56)}=28.8, p<$ 0.0001 ), and although no overall group effect was detected ( $p=$ 0.2 ), estrogen-replacement monkeys tended to score better than controls on a subset of delays (group by delay interaction: $F_{(4,56)}$ $=2.0, p=0.1$ ). Planned comparisons revealed the source of this marginal interaction; whereas the OVX-E and OVX-veh groups scored equivalently at the shortest and longest retention intervals, recognition accuracy was reliably superior among OVX-E monkeys at two delays of intermediate duration (unpaired $t$ tests; 30 sec delay: $t_{(14)}=-2.4, p<0.05 ; 120$ sec delay: $t_{(14)}=-2.2, p<$ $0.05)$. These findings suggest that estrogen modulation of cognitive aging in the monkey extends beyond DR and influences performance on a hallmark test of medial temporal lobe memory.

Next we considered the possibility that the behavioral consequences of estrogen replacement are secondary to changes in motivation, perceptual abilities, or other factors that could affect performance across multiple testing procedures that emphasize these capacities. Subjects were tested on a series of discrimination problems that required learning which of two visually distinct objects was consistently associated with reward (Fig. 1). The acquisition of rapidly learned object discriminations is impaired by extensive medial temporal lobe lesions (Zola-Morgan and Squire, 1985), but damage limited to the hippocampal formation has substantially less effect (Teng et al., 2000). Consistent with these observations, the subtle changes in medial temporal lobe function that accompany normal aging largely spare object discrimination learning and retention in monkeys (Rapp, 1990; Lai et al., 1995).

Estrogen- and vehicle-treated subjects acquired OD problems rapidly, scoring above $90 \%$ correct, on average, within three test sessions (Fig. 3) (repeated-measures ANOVA; main effect of trial block: $\left.F_{(8,112)}=54.8, p<0.0001\right)$. There was no indication of a hormone treatment effect at any point in training (main effect of group: $p>0.8$ ), including trials early in the course of learning that are especially sensitive to disruption by experimental medial temporal lobe damage and aging (Rapp, 1993; Teng et al., 2000). The conclusion from these findings is that a global estrogen influence on nonspecific performance factors fails to account for the benefits of treatment observed on the DR and DNMS tasks.

A review of our blinded protocol records revealed experimental treatment errors in two monkeys, involving 3 of the 251 injections provided over the course of the study. In one case a subject in group OVX-E was administered vehicle during DNMS testing with extended delays. However, this animal scored well within the range of other OVX-E subjects, suggesting that the cognitive effects of long-term estrogen replacement may persist after treatment. In the second case, another OVX-E monkey mistakenly received a vehicle injection toward the end of testing on the OD task (i.e., a procedure that proved insensitive to estrogen status). Neither the qualitative nor the statistical pattern of results was
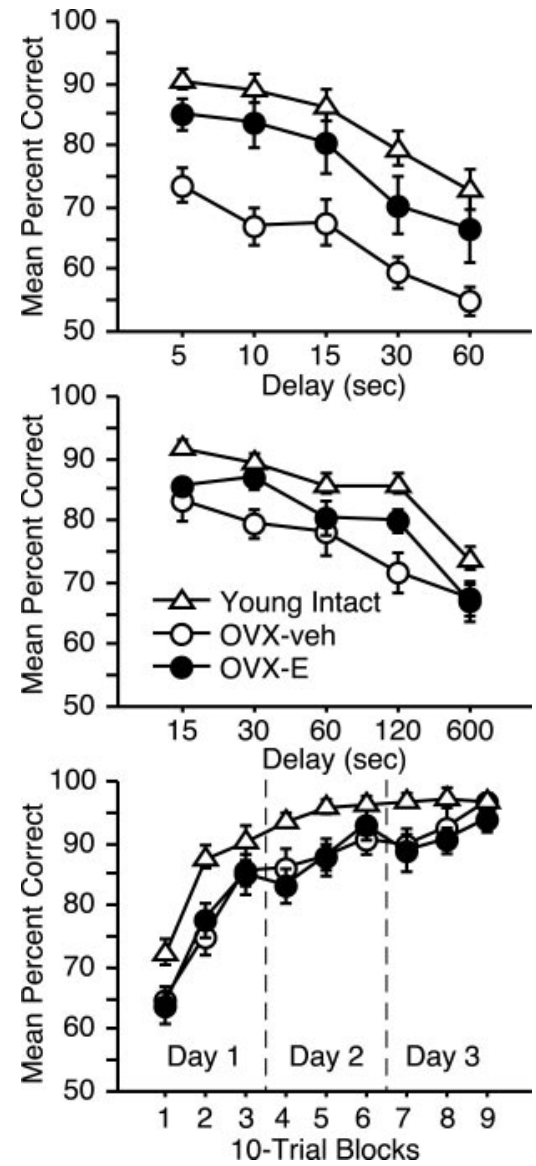

Figure 4. Neuropsychological performance in aged OVX-veh and OVX-E monkeys relative to young intact subjects. Top, Delay component of DR; middle, DNMS; bottom, OD, organized as in Figure 3. Error bars indicate SEM.

substantially affected when data from the two affected monkeys were excluded from consideration.

\section{Cognitive effects of OVX and estrogen in relation to performance in young monkeys}

To more fully characterize the nature and magnitude of ovarian hormone effects on cognitive aging, we compared performance in the aged OVX monkeys with findings from intact, young adults (mean age \pm SEM, 5.2 years \pm 5 months). Scores from the delay component of DR revealed an overall group difference (Fig. 4) (repeated-measures ANOVA; main group effect: $F_{(2,35)}=11.0$, $p<0.0005)$, and task accuracy in OVX-veh animals was impaired relative to both young monkeys (Bonferroni/Dunn; $p<0.0001$; critical adjusted $p$ value for post hoc comparisons $=0.017$ ) and aged OVX-E subjects $(p<0.015)$. However, performance in the latter groups was statistically equivalent $(p=0.11)$. These findings indicate that estrogen treatment can substantially reverse age-related cognitive impairment assessed by the DR task.

Parallel analysis revealed a modest benefit of hormone replacement on capacities measured by DNMS. Recognition accuracy differed across groups on the delay component of the task (Fig. 4) (main group effect: $F_{(2,32)}=8.6, p<0.005$ ), and this effect was attributable to impaired performance in group OVXveh relative to young controls $(p<0.0005)$. This result is similar to findings in intact monkeys demonstrating a modest but reliable age-related deficit on DNMS (Presty et al., 1987; Moss et al., 1988; Rapp and Amaral, 1991). Here, estrogen replacement par- 
tially restored recognition accuracy, and performance in group OVX-E was not reliably different from that in either young intact animals or age-matched OVX-veh monkeys ( $p$ exceeded the critical adjusted value for both comparisons).

Object-discrimination learning is resistant to age-related decline in intact monkeys, and the mild impairment that is sometimes observed is restricted to trials early in the course of acquisition (Rapp, 1993). Although this task failed to distinguish OVX-E and OVX-veh monkeys, both groups learned substantially more slowly than young animals overall (main group effect: $\left.F_{(2,32)}=6.2, p<0.01\right)$, and achieved normal asymptotic levels of accuracy only at the end of testing (Fig. 4, group-by-trial-block interaction $)\left(F_{(16,256)}=2.1 ; p<0.01\right)$. These findings suggest that OVX exacerbates age-related impairment in discrimination learning and that this effect is mediated by an estrogenindependent consequence of ovariectomy.

\section{Discussion}

The present study used a controlled, prospective design in monkeys to circumvent methodological constraints that have clouded the interpretation of research on the cognitive effects of HRT in postmenopausal women (Hogervorst et al., 2000). The results demonstrate that ovarian hormone status potently regulates cognitive aging in surgically menopausal subjects, including significant protective effects of low-dose, cyclic estradiol replacement as measured by two well characterized assessments, and an estrogen-insensitive exacerbation of age-related impairment on a third test. By comparison, investigations in young monkeys (Voytko, 2000, 2002; Lacreuse and Herndon, 2003), and in aged animals ovariectomized 10 or more years before treatment (Lacreuse et al., 2002), have reported substantially more circumscribed effects of ovarian hormone manipulation. For example, whereas estrogen replacement in younger animals was found to produce a transient improvement on a test of spatial attention, it failed to affect performance on a variant of the DR task that the present study revealed is robustly sensitive to estrogen status in aged monkeys (Voytko, 2000, 2002). Thus, available findings raise the possibility that ovarian hormone influences on cognitive function are age dependent, and that sensitivity to estrogen deficiency and replacement may increase around the time of menopause. Emerging evidence in rats supports the proposal that the behavioral and neurobiological response to estrogen replacement differs qualitatively in young and aged subjects (Adams et al., 2001; Markowska and Savonenko, 2002).

The tentative conclusion from a recent, comprehensive metaanalysis was that the cognitive effects of ovarian hormone replacement in women are not selective for a specific area of function and extend beyond the domain of memory (Hogervorst et al., 2000). In monkeys, manipulations that affect memory selectively tend to have an impact on DR and DNMS performance disproportionately at long retention intervals, when demands on recall and recognition are greatest (Zola-Morgan and Squire, 1985). Accordingly, the observation that the benefits of estrogen replacement were unrelated to the length of delay in these tasks is consistent with the proposal that they are mediated, at least in part, by an influence on other capacities. One possibility is that estrogen affects performance by modulating susceptibility to proactive interference and distraction. DR is especially sensitive in this regard because test sessions consist of many trials that are highly similar, in which the reward location is varied between only two possibilities. In DNMS, by comparison, new pairs of stimuli are presented on each trial and the potential for interference is primarily a consequence of feature overlap between ob- jects (e.g., shared color, texture, shape). Thus, the differential sensitivity of these tasks to estrogen replacement in aged OVX monkeys may correspond to their relative susceptibility to interference.

This account is compatible with the observation that $\mathrm{OD}$ is insensitive to estrogen status on the grounds that, in this task, the repeated presentation of a single object pair in each session may minimize the influence of interference and distraction. However, it should also be noted that ovariectomy per se (i.e., independent of hormone treatment condition) was associated with robust deficits in discrimination learning, the magnitude of which substantially exceeded the mild impairment sometimes found in intact aged monkeys (Rapp, 1990, 1993; Lai et al., 1995). By comparison, other manipulations that impair OD performance typically cause corresponding deficits in recognition memory, as assessed by DNMS (Zola-Morgan and Squire, 1985; Zola-Morgan et al., 1993; Teng et al., 2000). The pattern of results obtained here was qualitatively different, demonstrating that ovariectomy and estrogen replacement produce dissociable effects across these tasks. These findings imply that cognitive function during menopause may be influenced by multiple ovarian hormone changes and their interactions. Defining the synergistic and competitive effects of these changes on different information-processing capacities may be central to understanding the neuroendocrinology of cognitive aging.

Many brain regions outside the hypothalamic-pituitary axis are responsive to ovarian hormones, including multiple systems implicated in normal cognitive function (McEwen, 2002). Ovariectomy and hormone replacement in young adult monkeys influence the density of cholinergic and catecholaminergic fibers that innervate the dorsolateral prefrontal cortex (Kritzer and Kohama, 1998, 1999), for example, and related effects in other brain areas, including the hippocampus, have been reported in rats (Gibbs, 2000; Bowman et al., 2002). The potential functional significance of these effects is highlighted by evidence that the integrity of catecholamine input is critical for cognitive capacities mediated by the prefrontal cortex (Arnsten, 1998). Cognitive processing supported by the prefrontal cortex and hippocampal system is also vulnerable to age-related decline, and in some cases, these impairments are coupled with alterations in the same neurochemically specific ascending systems that are sensitive to ovarian-hormone manipulation (Gallagher and Rapp, 1997). Together, these findings raise the possibility that the broad cognitive effects we observed in the aged monkey may be mediated by the influence of ovariectomy and estrogen replacement on the widespread projections of subcortical modulatory systems.

Although the preceding account emphasizes the potential involvement of extrinsic inputs, gonadal steroids also affect the intrinsic circuit organization of the cortical targets of these projections (McEwen, 2002). Naturally occurring and experimental estrogen fluctuations potently regulate dendritic spine and synaptic density in the CA1 field of the rat hippocampus (Woolley et al., 1990; Woolley and McEwen, 1993), producing corresponding alterations in electrophysiological and metabolic measures of hippocampal activity (Woolley, 1999). Preliminary findings indicate that the same hormone-replacement regimen used here also induces spine proliferation in monkeys, in both the hippocampus and prefrontal cortex (Tang et al., 2002; Hao et al., 2003). In rats, these effects are at least partly dependent on NMDA receptors that mediate memory-related cellular plasticity in the hippocampus (Woolley and McEwen, 1994), and that themselves are regulated by estrogen (Gazzaley et al., 1996). Therefore, estrogen replacement at menopause might play a pro- 
tective role by promoting the maintenance of functional connectivity that is critical for cortical information processing. The present findings establish a nonhuman primate model for testing this proposal and evaluating other neurobiological consequences of ovarian hormone manipulation in relation to their effect on the cognitive outcome of aging.

CEE plus progestin formulations commonly prescribed in clinical practice in the United States have been optimized for the relief of hot flashes and related symptoms, but the effect of treatment on cognitive function in postmenopausal women remains controversial (Hogervorst et al., 2000). In addition, the safety of traditional HRT has been questioned on the basis of evidence documenting an increased risk for several negative outcomes, including stroke (Rossouw et al., 2002) and dementia (Shumaker et al., 2003). The replacement strategy adopted in the current study was different, consisting of unopposed estradiol injected according to a schedule that more closely mimicked normal estrogen dynamics in intact subjects. Although the specific variables responsible for the robust behavioral response we observed remain to be defined, one possibility is that dendritic spine growth and other neurobiological consequences of elevated estrogen may become refractory over the course of chronic treatment, and that cyclic replacement prevents desensitization. Emerging evidence also suggests that progesterone-related components of HRT can significantly modulate certain estrogen effects in monkeys, including both synergistic and antagonistic influences (Kritzer and Kohama, 1998, 1999) (McEwen, personal communication). Thus, an important direction for future research is to determine whether the cognitive benefits of unopposed estradiol reported here can be sustained when concurrent progesterone is provided at doses sufficient to offset the risk for endometrial cancer (Hale et al., 2002). Nonetheless, by documenting that low dose, cyclic replacement can broadly influence cognitive aging in surgically menopausal monkeys, our findings inform a risk/benefit analysis, encouraging the view that agerelated cognitive decline may be a tractable target in the development of novel treatment strategies.

\section{References}

Adams MM, Shah RA, Janssen WG, Morrison JH (2001) Different modes of hippocampal plasticity in response to estrogen in young and aged female rats. Proc Natl Acad Sci USA 98:8071-8076.

Arnsten AFT (1998) Catecholamine modulation of prefrontal cortical cognitive function. Trends Cogn Sci 2:436-447.

Bachevalier J, Mishkin M (1986) Visual recognition impairment follows ventromedial but not dorsolateral prefrontal lesions in monkeys. Behav Brain Res 20:249-261.

Bachevalier J, Landis LS, Walker LC, Brickson M, Mishkin M, Price DL, Cork LC (1991) Aged monkeys exhibit behavioral deficits indicative of widespread cerebral dysfunction. Neurobiol Aging 12:99-111.

Barrett-Connor E, Kritz-Silverstein D (1993) Estrogen replacement therapy and cognitive function in older women. JAMA 269:2637-2641.

Bowman R, Ferguson D, Luine V (2002) Effects of chronic restraint stress and estradiol on open field activity, spatial memory, and monoaminergic neurotransmitters in ovariectomized rats. Neuroscience 113:401-410.

Fuster J (2001) The prefrontal cortex-an update: time is of the essence. Neuron 30:319-333.

Gallagher M, Rapp PR (1997) The use of animal models to study the effects of aging on cognition. Annu Rev Psychol 48:339-370.

Gazzaley AH, Weiland NG, McEwen BS, Morrison JH (1996) Differential regulation of NMDAR1 mRNA and protein by estradiol in the rat hippocampus. J Neurosci 16:6830-6838.

Gibbs R (2000) Effects of gonadal hormone replacement on measures of basal forebrain cholinergic function. Neuroscience 101:931-938.

Gilardi KVK, Shideler SE, Valverde CR, Roberts JA, Lasley BL (1997) Characterization of the onset of menopause in the rhesus macaque. Biol Reprod 57:335-340.
Goldman-Rakic PS (1987) Circuitry of the primate prefrontal cortex and regulation of behavior by representational memory. In: Handbook of physiology (Mountcastle VB, Plum F, Geiger SR, eds), pp 373-417. Baltimore: Williams \& Wilkins.

Hale GE, Hughes CL, Cline JM (2002) Endometrial cancer: hormonal factors, the perimenopausal "window of risk," and isoflavones. J Clin Endocrinol Metab 87:3-15.

Hao J, Janssen WGM, Tang Y, Roberts JA, McKay H, Lasley B, Allen PB, Greengard P, Rapp PR, Kordower JH, Hof PR, Morrison JH (2003) Estrogen increases the number of spinophilin-immunoreactive spines in the hippocampus of young and aged female rhesus monkeys. J Comp Neurol, in press.

Hogervorst E, Williams J, Budge M, Riedel W, Jolles J (2000) The nature of the effect of female gonadal hormone replacement therapy on cognitive function in post-menopausal women: a meta-analysis. Neuroscience 101:485-512.

Korenman SG, Stevens RH, Carpenter LA, Robb M, Niswender GD, Sherman BM (1974) Estradiol radioimmunoassay without chromatography: procedure, validation and normal values. J Clin Endocrinol Metab 38:718-720.

Kritzer MF, Kohama SG (1998) Ovarian hormones influence the morphology, distribution, and density of tyrosine hydroxylase immunoreactive axons in the dorsolateral prefrontal cortex of adult rhesus monkeys. J Comp Neurol 395:1-17.

Kritzer MF, Kohama SG (1999) Ovarian hormones differentially influence immunoreactivity for dopamine beta-hydroxylase, choline acetyltransferase, and serotonin in the dorsolateral prefrontal cortex of adult rhesus monkeys. J Comp Neurol 409:438-451.

Lacreuse A, Herndon JG (2003) Estradiol selectively affects processing of conspecifics' faces in female rhesus monkeys. Psychoneuroendocrinology, in press.

Lacreuse A, Wilson M, Herndon JG (2002) Estradiol, but not raloxifene, improves aspects of spatial working memory in aged ovariectomized rhesus monkeys. Neurobiol Aging 23:589-600.

Lai ZC, Moss MB, Killiany RJ, Rosene DL, Herndon JG (1995) Executive system dysfunction in the aged monkey: spatial and object reversal learning. Neurobiol Aging 16:947-954.

Markowska AL, Savonenko AV (2002) Effectiveness of estrogen replacement in restoration of cognitive function after long-term estrogen withdrawal in aging rats. J Neurosci 22:10985-10995.

McEwen BS (2002) Estrogen actions throughout the brain. Recent Prog Horm Res 57:357-384.

Mishkin M (1978) Memory in monkeys severely impaired by combined but not by separate removal of amygdala and hippocampus. Nature 273:297-298.

Mishkin M, Pribram KH (1956) Analysis of the effects of frontal lesions in monkey. II. Variations of delayed response. J Comp Physiol Psychol 49:36-40.

Moss MB, Rosene DL, Peters A (1988) Effects of aging on visual recognition memory in the rhesus monkey. Neurobiol Aging 9:495-502.

O'Connell M (1995) Pharmacokinetic and pharmacologic variation between different estrogen products. J Clin Pharmacol 35:18S-24S.

O’Donnell KA, Rapp PR, Hof PR (1999) Preservation of prefrontal cortical volume in behaviorally characterized aged macaque monkeys. Exp Neurol 160:300-310.

Presty SK, Bachevalier J, Walker LC, Struble RG, Price DL, Mishkin M, Cork LC (1987) Age differences in recognition memory of the rhesus monkey (Macaca mulatta). Neurobiol Aging 8:435-440.

Rapp PR (1990) Visual discrimination and reversal learning in the aged monkey (Macaca mulatta). Behav Neurosci 104:876-884.

Rapp PR (1993) Neuropsychological analysis of learning and memory in the aged nonhuman primate. Neurobiol Aging 14:627-629.

Rapp PR, Amaral DG (1989) Evidence for task-dependent memory dysfunction in the aged monkey. J Neurosci 9:3568-3576.

Rapp PR, Amaral DG (1991) Recognition memory deficits in a subpopulation of aged monkeys resemble the effects of medial temporal lobe damage. Neurobiol Aging 12:481-486.

Resnick S, Maki P (2001) Effects of hormone replacement therapy on cognitive and brain aging. Ann NY Acad Sci 949:203-214.

Roberts JA, Gilardi KVK, Lasley BL, Rapp PR (1997) Reproductive senescence predicts cognitive decline in aged female monkeys. NeuroReport 8:2047-2051. 
Rossouw JE, Anderson GL, Prentice RL, LaCroix AZ, Kooperberg C, Stefanick ML, Jackson RD, Beresford SA, Howard BV, Johnson KC, Kotchen JM, Ockene J (2002) Risks and benefits of estrogen plus progestin in healthy postmenopausal women: principal results from the Women's Health Initiative randomized controlled trial. JAMA 288:321-333.

Sherwin B (2002) Estrogen and cognitive aging in women. Trends Pharmacol Sci 23:527-534.

Shideler SE, Munro CJ, Tell L, Owiti G, Laughlin L, Chatterton J, Lasley BL (1990) The relationship of serum estradiol and progesterone concentrations to the enzyme immunoassay measurements of urinary estrone conjugates and immunoreactive pregnanediol-3-glucoronide in Macaca mulatta. Am J Primatol 22:113-122.

Shumaker SA, Legault C, Thal L, Wallace RB, Ockene JK, Hendrix SL, Jones III BN, Assaf AR, Jackson RD, Morley Kotchen J, Wassertheil-Smoller S, Wactawski-Wende J (2003) Estrogen plus progestin and the incidence of dementia and mild cognitive impairment in postmenopausal women: the Women's Health Initiative Memory Study: a randomized controlled trial. JAMA 289:2651-2652.

Squire LR, Zola-Morgan S (1991) The medial temporal lobe memory system. Science 253:1380-1386

Tang Y, Janssen WGM, Hao J, Roberts J, McKay H, Hof PR, Morrison JH (2002) Effects of estrogen on dendritic spines in prefrontal cortex of young female rhesus monkeys. Soc Neurosci Abstr 28:368.12.

Teng E, Stefanacci L, Squire LR, Zola SM (2000) Contrasting effects on discrimination learning after hippocampal lesions and conjoint hippocampal-caudate lesions in monkeys. J Neurosci 20:3853-3863.
Tigges J, Gordon TP, McClure HM, Hall EC, Peters A (1988) Survival rate and life span of rhesus monkeys at the Yerkes Regional Primate Research Center. Am J Primatol 15:263-273.

Voytko ML (2000) The effects of long-term ovariectomy and estrogen replacement therapy on learning and memory in monkeys (Macaca fascicularis). Behav Neurosci 114:1078-1087.

Voytko ML (2002) Estrogen and the cholinergic system modulate visuospatial attention in monkeys (Macaca fascicularis). Behav Neurosci 116:187-197.

Woolley CS (1999) Electrophysiological and cellular effects of estrogen on neuronal function. Crit Rev Neurobiol 13:1-20.

Woolley CS, McEwen BS (1993) Roles of estradiol and progesterone in regulation of hippocampal dendritic spine density during the estrous cycle in the rat. J Comp Neurol 336:293-306.

Woolley CS, McEwen BS (1994) Estradiol regulates hippocampal dendritic spine density via an NMDA receptor-dependent mechanism. J Neurosci 14:7680-7687.

Woolley CS, Gould E, Frankfurt M, McEwen BS (1990) Naturally occurring fluctuation in dendritic spine density on adult hippocampal pyramidal neurons. J Neurosci 10:4035-4039.

Zola-Morgan S, Squire LR (1985) Medial temporal lesions in monkeys impair memory on a variety of tasks sensitive to human amnesia. Behav Neurosci 99:22-34.

Zola-Morgan S, Squire LR, Clower RP, Rempel NL (1993) Damage to the perirhinal cortex exacerbates memory impairment following lesions to the hippocampal formation. J Neurosci 13:251-265. 